Trascender, Contabilidad y Gestión Núm. 13 (enero - abril del 2020).

Universidad de Sonora. Departamento de Contabilidad.

ISSN: 2448-6388. Reserva de Derechos 04-2015-04172070800-203.

\title{
Decisiones políticas y rentabilidad, solvencia y endeudamiento del sector de energías renovables en España
}

Governmental policy decisions and profitability, solvency and indebtedness in the renewable energy sector in Spain

\author{
José Juan Déniz Mayor ${ }^{1}$; Ramón Maestre Valido ${ }^{2}$
}

Recibido: 18 de diciembre de 2019.

Aceptado: 11 de enero de 2020.

DOI: https://doi.org/10.36791/tcg.v13i0.78

JEL: M41. Contabilidad. M48. Política pública y regulación.

\begin{abstract}
Resumen
Se ha afirmado que la evolución del sector de energías renovables en España ha estado condicionada por la regulación vigente en cada momento, siendo el propósito del presente trabajo contrastar la validez de tal aserto. Así, partiendo de una muestra de 14.509 sociedades, dedicadas en exclusiva a la producción de electricidad mediante fuentes de energía renovable, se analiza si sus cifras de rentabilidad, solvencia y endeudamiento se ven afectadas por las normas dictadas al efecto, para el periodo 2001-2016.
\end{abstract}

Palabras clave: energía renovable, regulación, indicadores contables.

\section{Abstract}

It has been stated that the evolution of the renewable energy sector in Spain has been conditioned by the regulation in force at any given time, so the aim of this paper is to contrast the validity of this assertion. Thus, based on a sample of 14,509 companies, exclusively centered in the generation of electricity through renewable energy sources, it is analyzed if their figures of profitability, solvency and indebtedness are affected by certain rules approved for this purpose, across the period 2001-2016.

Keywords: renewable energy, regulation, accounting indicators.

\footnotetext{
${ }^{1}$ José Juan Déniz Mayor. Economista. Experto Contable Acreditado. Doctor en Ciencias Económicas y Empresariales. Profesor titular de Contabilidad Financiera y Organización Contable, en el Departamento de Economía Financiera y Contabilidad de la Universidad de Las Palmas de Gran Canaria (ULPGC). Correo: josejuan.deniz@ulpgc.es

${ }^{2}$ Ramón Maestre Valido. Graduado en Administración y Dirección de Empresas. Máster en Dirección Financiera y Contable de la Empresa, especialidad Finanzas Corporativas. Máster en Marketing y Comercio Exterior. Correo: rmaestrev@gmail.com
} 


\section{Introducción}

Los asuntos relacionados con el uso de fuentes de energía renovable han ido formando parte de la agenda de los principales partidos políticos españoles de manera paulatina, tal y como puede observarse consultando los distintos programas electorales, así como las acciones llevadas a cabo por aquellas fuerzas que se han encontrado al frente del Gobierno de la nación. Por tal motivo procede cuestionarse si el color político guarda relación con la situación y evolución del sector o si las decisiones adoptadas, bien para estimular la actividad, bien para acomodarla a la situación económica y presupuestaria del momento, producen algún efecto económico o financiero en las empresas.

En lo que respecta a España, la utilización de las energías renovables para la producción eléctrica ha experimentado un crecimiento continuado. A título ilustrativo y según la Secretaría de Estado de Energía (2017), dentro del mix energético, las distintas fuentes de energía renovable alcanzaron una participación del 13,9\% del consumo de energía primaria en el año 2016. Así, mientras el conjunto de las energías eólica, solar y geotérmica, suministró el 6,2\% de dicho consumo, el bloque formado por la biomasa, biocarburantes y residuos renovables contribuyó con otro 5,2\%. Finalmente, la energía aportada por los distintos medios hidráulicos ascendió a un 2,5\% del total.

A ello no han sido ajenas las denominadas políticas de primas (feed-in regulations), adoptadas por las diferentes administraciones con el propósito de estimular la producción de electricidad con origen renovable (Montoya et al., 2014). En general, dichas medidas (Couture et al., 2010) se basan en contratos a largo plazo en los que se ofrece un precio preferente por kWh (kilovatio-hora) generado, basado en el precio de mercado mayorista (feed-in premium), o un pago total por kWh (feed-in tariff), combinados con un acceso garantizado a la red de suministro, estando obligadas las compañías distribuidoras a adquirir la energía producida ${ }^{1}$.

En el presente trabajo se estudia la posible influencia de la regulación en el sector de producción de energías renovables entre 2001 y 2016. Así, se analizará si la promulgación de determinadas normas legales en la materia está relacionada con los cambios producidos en el valor de ciertas partidas e indicadores contables.

En la siguiente sección se expondrán las principales normas que han regulado el sector durante el periodo de estudio. En el tercer apartado se enuncian los objetivos, fuentes de datos, metodología de análisis y limitaciones, dedicándose el cuarto bloque a presentar y evaluar los resultados obtenidos. El trabajo finaliza con las principales conclusiones y la bibliografía citada.

\section{Regulación en España del sector de producción de energías renovables. Del impulso a la contención y los litigios}

En atención al horizonte temporal en que se enmarca el presente trabajo, parece oportuno comenzar el análisis histórico en el mes de diciembre de 1999, cuando el Plan de Fomento de las Energías Renovables en España (2000-2010) (Instituto de Diversificación y Ahorro Energético, IDAE, 1999) es aprobado por el Gobierno de España, en manos del Partido Popular (PP) en aquel momento. En dicho texto, que viene a desarrollar los compromisos recogidos en la Ley 54/1997, de 27 de noviembre, del Sector Eléctrico, se establecía como

\footnotetext{
${ }^{1}$ Frente a los modelos basados en primas, en los sustentados en certificados verdes negociables (tradable green certificates o TGC) los productores de electricidad con origen en renovables deben competir en dos mercados: uno general para todos los operadores, y otro basado en certificados verdes. Mientras en los sistemas de primas, las empresas se centran en la minimización de los costes internos, las políticas basadas en certificados introducen restricciones cuantitativas que contribuyen a determinar el precio de la electricidad de origen renovable (Jaraitė \& Kažukauskas, 2013).
} 
objetivo que a finales de 2010 las distintas fuentes de energías renovables suministrasen al menos un $12 \%$ del consumo de energía primaria, contando para ello con diferentes incentivos fiscales en función del tipo de tecnología utilizada, salvo en el caso de la eólica y la hidráulica, para las que se reservaban otros mecanismos como la armonización de requisitos de impacto ambiental. Además, mediante el Real Decreto 2818/1998, se daba a los productores basados en energías renovables la posibilidad de elegir entre dos tipos de incentivos: (a) una prima fija sobre el precio del mercado de la electricidad o (b) un precio total fijo, siguiendo así los enfoques feed-in.

No obstante, tales medidas de apoyo no fueron suficientes, pues entre 1999 y 2004 sólo se había logrado un $28,4 \%$ del objetivo global previsto. Tres fuentes habían mejorado hasta 2004: la eólica, los biocarburantes y el biogás. La minihidráulica había avanzado muy lentamente, mientras que la biomasa y la solar se desarrollaron por debajo de un ritmo óptimo. Según el Plan de Energías Renovables 2005-2010, aprobado en agosto de 2005 ya durante el mandato del Partido Socialista Obrero Español (PSOE), dos de las causas de este incumplimiento fueron, por un lado, el aumento de la demanda eléctrica, que se tradujo en un consumo de energía primaria y una intensidad energética muy por encima de lo esperado, y por otro, el incremento en el consumo de carburantes convencionales en el sector del transporte.

Por tal motivo, además de actualizar el contenido del plan anterior, manteniendo el objetivo de que las distintas fuentes renovables suministrasen el $12 \%$ del consumo de energía primaria, se incluyeron otros dos, basados en sendas propuestas recogidas en las
Directivas 2001/77/CE y 2003/30/CE, tales son: que el 29,4\% de la generación eléctrica fuese de origen renovable y que el 5,75\% del combustible utilizado en el transporte estuviese integrado por biocarburantes. Entre las acciones se encontraban medidas fiscales, de I+D+i y cambios legislativos. Para el primer propósito, relacionado además con el objeto del presente trabajo, el Gobierno consolidó a través del Real Decreto 661/2007 la figura de las Primas al Régimen Especial, comúnmente conocidas como "primas de las renovables”, justificadas por los beneficios que aportaban las energías renovables a la lucha contra el cambio climático. Estos incentivos consistían en subvenciones estatales que cubrían la diferencia entre los costes de generación utilizando fuentes renovables y los costes de generación bajo medios de producción convencional. Además, aquellos operadores que se acogieran a este régimen tendrían garantizado su acceso al sistema eléctrico de forma prioritaria. De acuerdo con Montoya et al. (2014), esto explicaría el salto experimentado entre 2007 y 2008, especialmente en el sector fotovoltaico que pasó de apenas unas escasas decenas de MW en los años previos, a casi 3.500 MW en 2008.

En su momento, los altos costes de esta medida no se consideraron relevantes, pues se estimaba que una economía como la española, con altas tasas de crecimiento, sería capaz de sufragar una forma de generación de energía que, en aquel entonces, ni técnica ni económicamente, se consideraba viable a largo plazo. Así, en el primer caso, tal medida de estímulo se tradujo en una sobredimensión de potencia instalada, llegándose a subvencionar instalaciones que estaban paradas. Desde un punto de vista económico, contribuyó a aumentar el denominado "déficit de tarifa", pues las primas no se

\footnotetext{
${ }^{1}$ Diferencia entre la cantidad total recaudada (integrada por los peajes de acceso, que son los pagos que los clientes deben realizar a los productores por el uso de las redes de transporte y distribución de la electricidad, que es fijado por el Gobierno, y ciertos cargos regulados por la Administración y pagados por los usuarios) y los costes reales asociados a las tarifas. Los peajes son fijados anualmente por el Ministerio con competencias en materia de energía.
} 
cargaban al déficit público estatal, como se hacía en otros países del entorno que también habían apostado por apoyar las energías renovables, sino que se convertían en un pasivo para el Estado en favor de las empresas productoras; si bien es verdad que la figura del déficit de tarifa ya tenía un cierto recorrido, tras la aprobación de la Ley 54/1997, que buscaba la liberalización del mercado de la energía y ya acumulaba los costes de la moratoria nuclear ${ }^{1}$. En España, las primas iban con cargo a los peajes de la tarifa. A pesar de que, a medida que se desarrollaban las tecnologías relacionadas con las energías renovables, sus costes se reducían, este hecho no se traducía en una disminución de las primas.

Este paisaje de subvenciones y sobredimensión recibió el nombre de burbuja verde, afectando fundamentalmente al sector fotovoltaico. Entre los principales motivos por los que no fue posible prever y controlar la acelerada expansión de las plantas basadas en esta tecnología, cabe citar su fácil y rápida instalación, mediante módulos, y que sus costes fuesen cada vez menores (Lomas et al., 2018; López, 2018), en comparación con las restantes alternativas. Esto explica que resultase atractivo para muchos pequeños $\mathrm{y}$ medianos inversores, además de los propietarios de terrenos, que, ante la desaceleración del mercado de la vivienda, buscaban nuevas oportunidades de negocios, máxime si éstos gozaban de incentivos gubernamentales. Mir-Artigues et al. (2015) afirman que un problema añadido fue la existencia de asimetrías informativas en favor de los productores, pues eran estos quienes conocían con precisión los costes de la tecnología aplicada y podían tener un incentivo para inflar los precios y obtener una mayor remuneración. Según estos autores, los costes unitarios para apoyar a los productores fotovoltaicos habían pasado de 39 euros/Mwh en 2007 a 242 euros/MWh en 2009; en cifras absolutas, de 194 millones de euros en 2007 a 2.629 millones de euros en 2009.

Así, en el Informe de evaluación del Plan de Energías Renovables (2005-2010) (Agencia Estatal de Evaluación de las Políticas Públicas y la Calidad de los Servicios, 2011, p.101) se reconocería que, si bien la política de estímulo a la energía eólica funcionó correctamente, el principal error en el caso de la gestión de la tecnología fotovoltaica fue la cuantiosa prima que se daba a los productores, pensada para el lanzamiento e impulso de esta forma de generación, pero no para una situación real en la que ya se habían sobrepasado los objetivos recogidos en el Plan de Energías Renovables. En el caso de la eólica, la normativa era eminentemente autonómica (salvo en la eólica off-shore, en la que se veían implicadas diferentes administraciones), lo que obligó a aprobar el Real Decreto 1028/2007, para delimitar las competencias administrativas entre los distintos Ministerios, según procediese, para otorgar la autorización pertinente.

Nuevamente, procede hacer referencia al citado déficit tarifario que sólo para el ejercicio 2008 ascendió a 5.819 millones de euros, según la memoria de la Comisión Nacional de la Energía (2009). Teniendo en cuenta que en esos momentos España ya estaba en plena crisis financiera, se aprueba el Real Decreto-ley 6/2009 cuyo objetivo era establecer unos límites que acotasen este crecimiento del déficit de tarifa, pero a la vez implantar un bono social que protegiese la seguridad jurídica y confianza de los consumidores más desprotegidos. En lo que respecta a las medidas tomadas para disminuir el déficit, se estableció, entre otras, la creación de un registro de pre-asignación de retribución, que permitiera

\footnotetext{
1 Aunque el propósito de la Ley 54/1997 era iniciar un proceso de liberalización en las actividades de producción, distribución y comercialización de la electricidad en España, creándose un mercado abierto donde los consumidores fuesen libres de elegir sus suministradores de energía, parece conveniente cuestionarse si la noción de déficit tarifario no cuestiona el objetivo principal de la liberalización y es que a través de los precios del mercado se consiguiese una mejor comprensión en torno al precio de un bien escaso como es la energía, lo que debería contribuir a fomentar el ahorro y la eficiencia energética.
} 
conocer las instalaciones proyectadas y verificar el cumplimiento de las condiciones para ejecutarse $\mathrm{y}$ acceder al sistema eléctrico con todos los requisitos legales y reglamentarios, el volumen de potencia asociado a las mismas, así como el impacto en los costes de la tarifa eléctrica y su calendario. La razón de ello es que dada la tendencia que seguían estas tecnologías, se podía poner en riesgo en el corto plazo la sostenibilidad económica y técnica del sistema.

Posteriormente y con la aprobación del Real Decretoley 14/2010, con el objetivo de corregir el déficit tarifario, se adopta entre otras decisiones la limitación, con efectos retroactivos, de horas equivalentes de funcionamiento de instalaciones fotovoltaicas a los efectos de retribución, tomando como base el cociente entre la producción neta anual expresada en kWh y la potencia nominal de la instalación expresada en $\mathrm{kW}$. Según Montoya et al. (2014), solo en el sector fotovoltaico, tales cambios habrían causado pérdidas cercanas al 30\% de los ingresos esperados, en 2011, 2012 y 2013, reduciéndose al 10\% después de 2014. También en 2010, se aprueba el Real Decreto 1003/2010, que busca regular la liquidación de la prima en las instalaciones de producción de tecnología fotovoltaica en régimen especial, estableciéndose un procedimiento de acreditación de unas condiciones que, necesariamente, debían cumplirse para que los interesados optasen a las primas.

En 2011 se aprueba la Ley 2/2011 de Economía Sostenible, que incluye en su contenido la promoción de las energías renovables, la reducción de emisiones y residuos, así como el fomento del ahorro y la eficiencia energética, prolongándose el plazo de percepción de retribución primada por el uso de plantas fotovoltaicas, lo que aparentemente contradice las medidas de contención dictadas previamente.

También durante 2011 se aprueba el Plan de Energías Renovables 2011-2020 (IDAE, 2011), acorde con los objetivos de la Unión Europea establecidos en la Directiva 2009/28/CE para el año 2020, consistentes en una cuota mínima del $20 \%$ de energía procedente de fuentes renovables en el consumo final bruto de energía y una cuota mínima del 10\% de energía procedente de fuentes renovables en el consumo de energía en el sector del transporte.

Tras las elecciones de noviembre de ese año, en las que el Partido Popular obtiene la mayoría absoluta tanto en el Congreso de los Diputados como en el Senado, entre las primeras medidas adoptadas, se encuentra la aprobación del Real Decreto-ley 1/2012, por el que se suspenden los procedimientos de preasignación de retribución, así como los incentivos económicos para nuevas instalaciones de producción de energía eléctrica usando fuentes renovables. Según el nuevo gobierno, debido al elevado déficit, tanto de tarifa como público, era necesario buscar otro modelo de retribución que no pusiera en riesgo el sistema entero. Esta medida sólo afectaba a las nuevas instalaciones, no a las ya instaladas ni aquellas que ya estuvieran inscritas en los preregistros, ni tampoco influiría en los objetivos establecidos por el Plan de Energías Renovables 20112020.

Ese año también se aprueba la Ley 15/2012, que establece la financiación de los costes del sistema tanto por los ingresos propios del mismo, como por las partidas correspondientes de los Presupuestos Generales del Estado. Para ello, se crea el Impuesto sobre el valor de la producción de energía eléctrica que afecta a todos los productores de régimen, nuevamente con el objetivo 
de contribuir a la sostenibilidad del sistema ${ }^{1}$. Al año siguiente y debido a la todavía continuada situación de déficit, se aprueba el Real Decreto-ley 2/2013, que fija un nuevo método de cálculo de las retribuciones, tarifas y primas, con el objetivo de reducir los costes y evitar que los consumidores asuman dicha carga. En el mes de julio de ese mismo año, se aprueba el Real Decreto-ley 9/2013, con el que se sigue buscando garantizar la estabilidad financiera del sistema eléctrico. Este Real Decreto-ley, que suprime de facto el régimen especial ${ }^{2}$, habilita al Gobierno a aprobar un nuevo marco jurídico y económico para las instalaciones de producción de energía eléctrica existentes a partir de energía renovable, cogeneración y residuos. Esta decisión pretende dar cobertura a los costes adicionales que estas instalaciones soportan, respecto al resto de tecnologías en el mercado. Asimismo, se realiza una reasignación de los costes imputables al sistema eléctrico, pues no tendrán tal consideración aquellos que no vengan determinados por normas que no sean de aplicación en todo el territorio nacional y que deberán responder exclusivamente a la actividad de producción de energía eléctrica.

En diciembre es aprobada la Ley 24/2013, en cuyo artículo 14.7 se establece que el Gobierno podrá establecer un régimen retributivo especial para fomentar la producción de energías renovables cuando exista la obligación de cumplir algún objetivo derivado de Directivas $\mathrm{u}$ otras normas europeas o cuando la implementación del mismo conlleve una reducción del coste energético y de dependencia exterior. Ahora bien, el propio articulado establece que, con carácter general, las instalaciones de autoconsumo estarán obligadas a contribuir a la financiación de los costes y servicios del sistema en la misma cuantía que el resto de los consumidores, aunque en el artículo 9.3 y en la disposición transitoria novena, se prevén excepciones para los casos en los que el autoconsumo suponga una reducción de costes para el sistema y, transitoriamente hasta el 31 de diciembre de 2019, para las instalaciones existentes de cogeneración.

En desarrollo de dicha Ley se aprobará el Real Decreto 413/2014, que regula la actividad de producción de energía eléctrica a partir de fuentes renovables, cogeneración y residuos, cuyo objeto es la regulación del régimen jurídico y económico de esta actividad. En este sentido, cabe destacar la reforma del sistema de incentivos, donde sólo aquellas instalaciones que no hayan superado su respectiva vida útil seguirán recibiendo primas. De igual modo se establecen los derechos y las obligaciones de este tipo de instalaciones y se regula su participación en el mercado eléctrico. Asimismo, se dicta la Orden IET/1045/2014, por la que se establecen los parámetros retributivos de las instalaciones incluidas en la disposición adicional primera del Real Decreto anterior.

Por su parte, el Real Decreto-ley 9/2015 abordará, de forma excepcional y siempre que se garantice la seguridad y la sostenibilidad económica y financiera del sistema y con las condiciones que el Gobierno regule, ciertas reducciones de peajes, cargos y costes para determinadas categorías de consumidores de baja tensión de la modalidad de suministro con autoconsumo

\footnotetext{
${ }^{1}$ No obstante, a mediados de 2016 el Tribunal Supremo y mediante sendos Autos de 14/06/2016 (Tribunal Supremo, 2016a, 2016b), plantea ante el Tribunal Constitucional una cuestión de inconstitucionalidad contra determinados artículos de dicha ley, entre ellos el relativo al Impuesto sobre la producción de electricidad al considerar que el hecho imponible ya está gravado por otro tributo (el Impuesto sobre Actividades Económicas).

${ }^{2}$ Esto implicaba además que en territorios desconectados de la red peninsular, como las islas Canarias, se perdiese el derecho a la prima, quedando sometidas al sistema retributivo nacional basado en una subasta diaria de energía o pool, a pesar de operar sin conexión con el territorio continental y sin tener en cuenta que los costes reales de generación basados en fuentes convencionales eran menos competitivos en el archipiélago (Mesa, 2015, 05 de mayo).
} 
(siempre que la potencia máxima contratada de consumo como la instalada de generación no sean superiores a 10 kW); mientras que el Real Decreto 900/2015 desarrollará los aspectos relativos a las condiciones administrativas, técnicas y económicas, estableciendo el pago de peajes de respaldo a cargo del autoconsumo energético $^{1}$, para contribuir a la cobertura de los costes de distribución y mantenimiento de la red, así como el compromiso de los autoproductores de verter su excedente de producción gratuitamente a la red, si la instalación es de menos de 100 kW.

En atención a lo expuesto, es factible sostener que la existencia de cambios normativos a lo largo del periodo de análisis puede haber dificultado el alcanzar un nivel adecuado de estabilidad y seguridad jurídica, con el consiguiente impacto en el sector de las energías renovables, al oscilar desde la adopción de medidas de estímulo hasta el establecimiento de acciones de contención, algunas de ellas consideradas retroactivas ${ }^{2}$. Así, desde una primera etapa basada en la figura de las primas a las energías renovables, apoyada en el Real Decreto 661/2007, se llega al Real Decreto-ley 9/2013, que suprime de facto el régimen especial $\mathrm{y}$, como colofón, al Real Decreto 900/2015 y el "Impuesto al sol”. Tal es la posición de Montoya et al. (2014), quienes afirman que la producción de energía mediante fuentes renovables ha llegado a experimentar una crisis y una incertidumbre legal de tal magnitud que España habría dejado de ser uno de los países más relevantes en el campo fotovoltaico, mientras que, en la eólica, aunque seguía formando parte de los cuatro países más importantes del mundo, su desarrollo se paralizaría durante los siguientes años.

Prueba de ello, es que durante la primera mitad de 2017 el Gobierno de España perdiese el primer laudo internacional en el Centro Internacional para el Arreglo de Diferencias sobre Inversiones (CIADI), dependiente del Banco Mundial, frente a Eiser Infrastructure Limited, por los recortes que había sufrido el sector desde 2010. En este caso, el CIADI dictaminó que el Gobierno debía pagar una multa de 128 millones de euros, siendo ésta, la primera sentencia de las casi treinta demandas que acumulaba España en este organismo internacional (Noceda, 2017, 5 de mayo; “España pierde su primer arbitraje”, 2017, 5 de mayo). No obstante, entre los dictámenes más recientes en el momento de redactar estas líneas, destaca el emitido a favor del Estado euros ("España sigue su racha y gana otro laudo renovable (132 millones) por unanimidad, 2020, 11 de marzo).

\section{Aspectos metodológicos}

\section{Objetivos}

Dado lo expuesto anteriormente, y en referencia a las empresas del sector de generación de electricidad bajo fuentes de energía renovables, el objetivo del presente trabajo es determinar si la producción normativa afecta a la rentabilidad, solvencia y endeudamiento de estas compañías.

\footnotetext{
${ }^{1}$ Dicho gravamen, conocido también como “Impuesto al sol”, abarca un cargo por potencia, tanto si la instalación de producción posee más más de $100 \mathrm{~kW}$ o si se utilizan acumuladores para conservar el excedente de energía producida; y otro por la diferencia entre toda la energía producida y el excedente volcado a la red general. El Real Decreto establece un régimen sancionador para quienes no hayan regularizado sus instalaciones que abarca desde los 6 millones a los 60 millones de euros. Aunque, como señalan Aragonés et al. (2016), debe tenerse en cuenta que, en el sistema legal español, las sanciones deben ser proporcionales al impacto económico de la infracción y los ingresos de la persona sancionada, y que por tanto tales cifras están muy lejos de lo que podría aplicarse en la práctica, no deja de ser llamativo el importe mínimo teóricamente aplicable.

2 El problema de los cambios legales retrospectivos y retroactivos, como medidas de contención ante decisiones previas de apoyo a las energías renovables, no es exclusivo de España. Otros países se encuentran en similar situación (véase Fouquet y Nysten, 2015).
} 


\section{Selección de la muestra}

Para seleccionar la muestra de empresas para el estudio se utilizó la base de datos SABI (Sistema de Análisis de Balances Ibéricos) propiedad de la empresa Informa S.A., y gestionada por Bureau van Dijk Electronic Publishing. Esta base de datos recoge informes económicos y financieros de más de un millón de empresas en España.

El criterio de selección se basó en la actividad principal desarrollada por las empresas a analizar, la producción de electricidad de origen renovable, debiendo estar inscritas en España, durante el periodo de análisis, el cual abarcaba de 2001 a 2016. A tal objeto se recurrió a la Clasificación Nacional de Actividades Económicas (CNAE), aprobado por Real Decreto $475 / 2007^{1}$, centrándose en las sociedades cuyos códigos primarios fuesen los siguientes:

\section{Producción de energía hidroeléctrica}

3518. Producción de energía eléctrica de origen eólico

3519. Producción de energía eléctrica de otros tipos

De acuerdo con tal criterio, el resultado inicialmente obtenido tras una búsqueda realizada el 18 de junio de 2018, fue de 18.383 empresas. Ahora bien, es factible que las compañías de mayor tamaño se caractericen por la diversificación de sus medios de producción de energía, abarcando no solo las fuentes renovables sino también las basadas en combustibles fósiles y nucleares. Además, puede suceder que empresas orientadas a la distribución y comercialización de electricidad o incluso empresas instaladoras de, por ejemplo, placas solares (que son ajenas al sector) se atribuyan erróneamente la actividad de producción. Por tal motivo, fue necesario identificar aquellas empresas que tuviesen como códigos primarios de la CNAE 2009 los siguientes:
3512. Transporte de energía eléctrica.

3513. Distribución de energía eléctrica

\section{Comercio de energía eléctrica}

3516. Producción de energía eléctrica de origen térmico convencional

3517. Producción de energía eléctrica de origen nuclear

Se localizaron 1.843 empresas que no necesariamente coincidían con alguna o algunas de las compañías de la muestra inicial. Asimismo, se consideró si las actividades de cogeneración (aquellas que generan en un mismo proceso energía eléctrica y calor útil) podrían entrar dentro de la categoría de energías renovables, pero como su fundamento básico es la energía fósil se decidió no incorporarlas al estudio (se identificaron 750). Similar decisión se adoptó con las empresas dedicadas a los biocombustibles (se detectaron 157). En estos dos casos, la búsqueda se basó en el objeto social declarado, así como en la denominación social de la entidad, llegándose a realizar consultas en sus páginas de internet en caso de duda. Tras la comparativa entre unas y otras, se decidió descartar a 345 empresas.

Dado que el estudio se realizó sobre empresas que han llevado a cabo actividades en el sector, se realizó un cribado adicional para seleccionar sólo aquellas sociedades que declararon tener una cifra de negocios superior a 0 euros (como indicador de la existencia de actividad), en algún momento del periodo de análisis, ascendiendo a 14.531 compañías. Finalmente se hicieron pruebas de consistencia de datos, tales como verificar la ecuación de equilibrio contable, existencia de patrimonio contable, etc., eliminándose 22 observaciones. Ello hizo que la muestra final quedase integrada por 14.509 empresas.

\footnotetext{
${ }^{1}$ Si bien con la clasificación NACE Rev 2 (2006) se llega a distinguir de forma exhaustiva las distintas modalidades de producción a partir de energías renovables, la base de datos SABI no opera con este nivel de desglose.
} 


\section{Hipótesis}

En el presente estudio se propone como hipótesis a contrastar, en relación con las empresas del sector de producción de energías renovables:

a) La rentabilidad económica, la rentabilidad financiera, la solvencia y el endeudamiento cambian tras la aprobación en 2007 del Real Decreto 661/2007, gracias al cual se implantaron las primas a la producción de energía renovable.

b) La rentabilidad económica, la rentabilidad financiera, la solvencia y el endeudamiento cambian tras la aprobación en 2013 del Real Decreto-ley 2/2013, el Real Decreto-ley 9/2013, y la Ley 24/2013, que conllevaron, entre otras medidas, la desaparición de facto del régimen especial.

\section{Metodología de análisis}

Se efectuó, en primer lugar, un análisis financiero comparativo de los agregados de las partidas contables de total activo, fondos propios, ingresos de explotación y resultado del ejercicio, así como de las ratios de rentabilidad económica, rentabilidad financiera, solvencia a corto plazo y endeudamiento ${ }^{1}$; para, posteriormente, llevar a cabo un estudio estadístico univariante que permitiese obtener pruebas que contrastasen las hipótesis anteriormente indicadas, en relación con la rentabilidad económica, la rentabilidad financiera y las ratios de solvencia y endeudamiento. A tal efecto, las variables seleccionadas fueron sometidas al test no paramétrico de Wilcoxon-Mann-Whitney para muestras pareadas, comparando las empresas comunes en los periodos de contraste, es decir, aquellas que realizaron operaciones tanto antes como después del hecho analizado. Para esta prueba no hizo falta excluir los valores extremos, pues el propio test reduce el impacto de los mismos al operar con los valores ordinales y no con las observaciones originales. En el caso concreto de las rentabilidades financiera $\mathrm{y}$ económica, además se estudió el cambio de signo (de negativo a positivo y viceversa), aplicando la prueba $\chi^{2}$ de Pearson. La aplicación utilizada fue STATA 11/1 (StataCorp LP, 2009).

\section{Limitaciones}

La principal limitación del estudio se encuentra en el cambio de los formatos de las cuentas anuales utilizados para la obtención de los datos con los que llevar a cabo el estudio. Dado que el horizonte temporal del trabajo abarca desde 2001 hasta 2016, la modificación de la regulación contable afecta al mismo, pues hasta 2007 las empresas se regían por el Plan General de Contabilidad de 1990. Sin embargo, a principios de 2008 entró en vigor un nuevo Plan General de Contabilidad, basado en las normas del International Accounting Standards Board (IASB) adoptadas por la Unión Europea, así como una versión reducida del mismo para pequeñas y medianas empresas. Una forma de evitar esta limitación consistió en utilizar el formato global de cuentas de la aplicación SABI, asumiendo que las partidas que éstas muestran bajo distintas regulaciones contables se presentan en un formato homogéneo $\mathrm{y}$, por ende, comparable.

Cabe destacar el caso de las empresas con fondos propios negativos, pues determinadas magnitudes contables como la rentabilidad financiera podrían aparecer en negativo aun teniendo resultados positivos o una empresa que registrase pérdidas podría figurar con una rentabilidad positiva, provocando problemas de

\footnotetext{
${ }^{1}$ La ratio de rentabilidad financiera se calculó como cociente entre el resultado del ejercicio y los fondos propios; la ratio de rentabilidad económica como cociente entre el resultado antes de intereses y el total activo; la ratio de solvencia como cociente entre el activo corriente y el pasivo corriente; y la ratio de endeudamiento como cociente entre el pasivo y los fondos propios.
} 
inconsistencia. Por ello, se decidió excluir del cálculo de la rentabilidad financiera a aquellas empresas que, durante el periodo de estudio, poseyesen fondos propios negativos, conservándose las mismas para el resto de los análisis.

Otro caso singular es el de las empresas con pasivo corriente nulo o casi nulo. Bajo tal supuesto, el valor de la ratio de solvencia a corto plazo es infinito o tan elevado que distorsiona el análisis del conjunto. Por tal motivo, se decidió excluir del cálculo de dicha ratio aquellas observaciones en las que se produjese tal incidencia lo cual, sumado al hecho de que en la muestra existen empresas sin partidas corrientes, hace que el número de datos considerados sea menor que en el caso de las rentabilidades y el endeudamiento.

Finalmente, cabe mencionar que los datos utilizados se presentan en términos nominales sin deflactar, por lo que no se ha tenido en cuenta el posible impacto de la inflación.

\section{Presentación y análisis de los datos}

En este apartado se expondrá, en primer lugar, un análisis descriptivo del sector de producción de electricidad por fuentes renovables desde el año 2001 hasta 2016. Seguidamente, se evaluará el posible impacto del signo político del Gobierno, así como de determinadas normas legales, sobre ciertas variables contables, en concreto la rentabilidad, la solvencia y el endeudamiento, de las empresas que estuvieron activas tanto antes como después del hecho considerado.

\section{Datos básicos del sector}

Como se puede observar en la Tabla 1, con datos referidos a 2001, 2014 (ejercicio con mayor número de empresas inscritas) y 2016, en el primer año de análisis los activos medios, así como la mediana y el percentil 75 , son superiores a los restantes ejercicios, lo que denota la existencia de grandes empresas, en contraste con 2014 y 2016, donde a pesar del mayor número de compañías participantes, tales valores son inferiores, indicativos de la atomización del sector. Observaciones similares cabe realizar con respecto a las restantes variables consideradas. Es reseñable que en 2014 el resultado contable global del sector sea negativo y que no todas las empresas inscritas tuvieron actividad.

Tabla 1. Estadística descriptiva básica años 2001, 2014 y 2016 (en miles de euros)

\begin{tabular}{lrcrrr}
\hline \multicolumn{1}{r}{ Variable } & N & Suma & Media & Mediana & Percentil 75 \\
\hline Activo 2001 & 881 & $15.243 .422,00$ & $17.302,41$ & $1.130,66$ & $6.089,42$ \\
Activo 2014 & 11.011 & $63.993 .827,40$ & $5.811,81$ & 460,35 & 703,82 \\
Activo 2016 & 9.913 & $57.896 .852,20$ & $5.840,50$ & 390,6 & 635,63 \\
Fondos propios 2001 & 881 & $6.095 .855,68$ & $6.919,25$ & 351,76 & $1.564,43$
\end{tabular}


Tabla 1. Continuación...

\begin{tabular}{lrrrrr}
\hline \multicolumn{1}{r}{ Variable } & N & \multicolumn{1}{c}{ Suma } & Media & Mediana & Percentil 75 \\
\hline Fondos propios 2014 & 11.011 & $10.226 .245,40$ & 928,73 & 20,36 & 145,28 \\
Fondos propios 2016 & 9.912 & $15.360 .232,60$ & $1.549,66$ & 32,27 & 168,44 \\
Ing. explotación 2001 & 722 & $2.919 .654,30$ & $4.043,84$ & 442,2 & $1.784,15$ \\
Ing. explotación 2014 & 10.705 & $12.236 .031,20$ & $1.143,02$ & 62,4 & 88,36 \\
Ing. explotación 2016 & 9.738 & $10.957 .549,60$ & $1.125,24$ & 66,27 & 83,17 \\
Resultado 2001 & 844 & $536.240,88$ & $.635,36$ & 6,96 & 110,5 \\
Resultado 2014 & 10.878 & $-818.976,59$ & $-75,29$ & $-2,79$ & 7,45 \\
Resultado 2016 & 9.799 & $585.979,66$ & 59,8 & 1,55 & 9,87 \\
\hline
\end{tabular}

Fuente: Elaboración propia a partir de SABI @ , 2017.

En el Gráfico 1 se presenta la evolución del sector atendiendo al número de sociedades inscritas para cada uno de los años.

Destaca cómo, a partir de 2008 y en aparente coincidencia con la aprobación del Plan de Energía Renovables 2005-2010 y el RD 661/2007, se produce un escalada en el número de sociedades inscritas que se estabilizará en 2014, quizás como consecuencia de la aprobación de los Reales Decretos-leyes 2/2013 y 9/2013, considerados restrictivos para el sector. Así, mientras en 2001 el número de sociedades fue de 881, en 2014 la cifra era de 11.011 para bajar hasta 9.913 en 2016. López (2018) señala, a este respecto, la singular estructura de propiedad del modelo español cuando se analiza el sector fotovoltáico, caracterizado por grandes instalaciones, divididas en pequeñas unidades de explotación, al objeto de minimizar costes mientras se obtenían las mayores primas por pequeñas instalaciones ${ }^{1}$.

\footnotetext{
${ }^{1}$ Según dicho autor, dicho modelo habría supuesto un sobrecoste para el sistema de 373 millones de euros, solo en 2008, equivalente al $32,4 \%$ de los ingresos totales de los productores fotovoltaicos. 
Gráfico 1. Evolución del número de empresas del sector

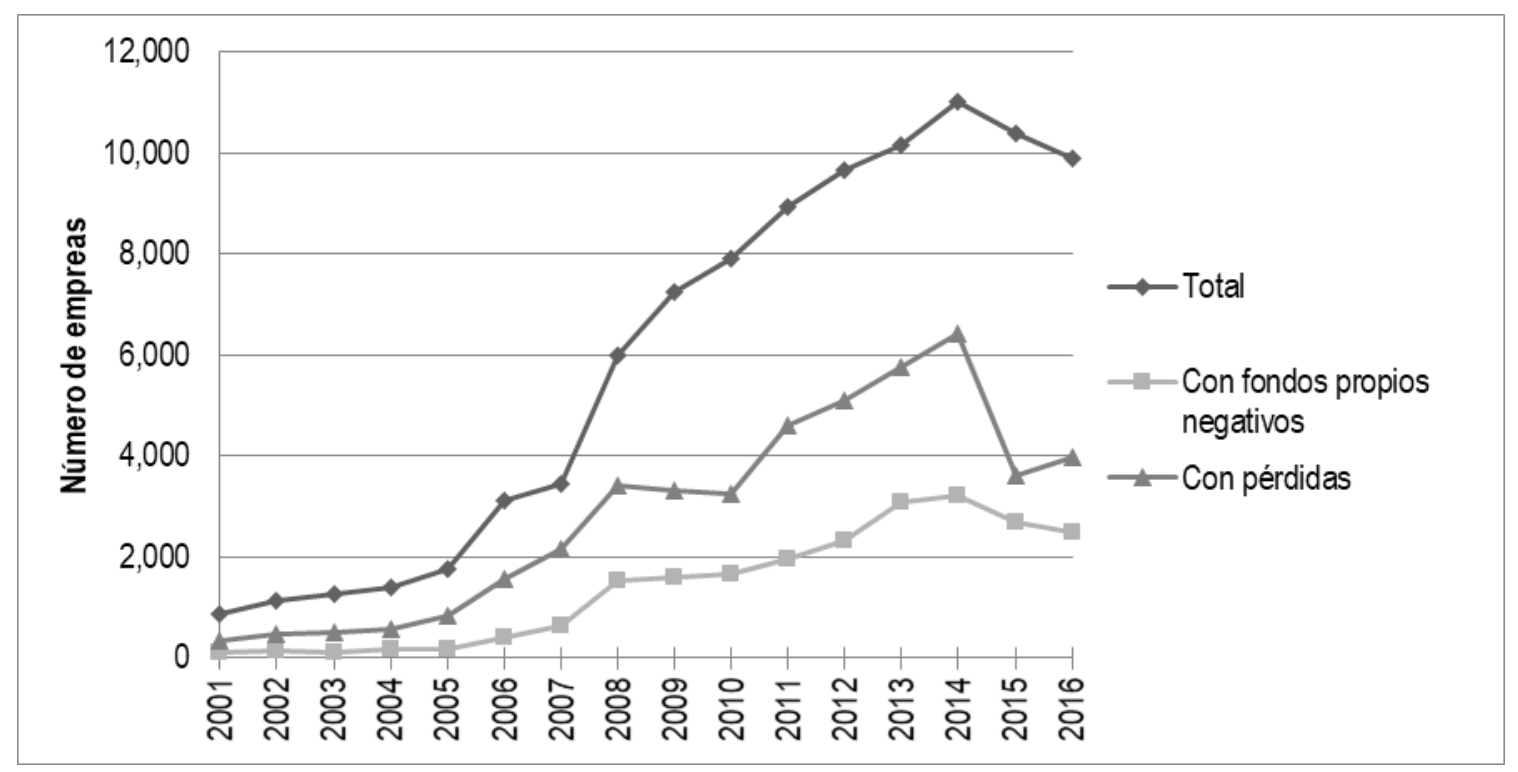

Fuente: Elaboración propia a partir de SABI @ , 2017.

A efectos ilustrativos cabe citar el caso de las empresas con fondos propios negativos: en 2001 la cifra fue de 89 (10,10\% del total) y en 2016 de 2.484 (25,06\% del total), alcanzándose el mayor porcentaje en 2013, con 3.089 compañías (un 30,39\% del total). Por su parte, la cuantía de empresas con pérdidas a finales de 2016 fue de 3.987 (40,22\% del total), pudiendo ser sintomático de una actividad en crisis, si bien cabe reseñar que fue en 2007 cuando se alcanzó el mayor porcentaje de empresas que se encontraban en tal situación (62,71\% del total), para empezar a decaer a partir del año siguiente, con el incremento en el número de empresas inscritas, y volver a crecer en 2011, llegándose a la cota del 58,29\% en 2014.

En lo que respecta al total activo del sector, a finales de 2001 su valor era de 15.243 millones de euros, mientras que a finales de 2016 esta cifra ascendía a 57.897 millones de euros, lo que supone una variación del 280\%. Sin embargo, fue en 2010 cuando el volumen de activo alcanzó su punto más alto tal y como se observa en el Gráfico 2, situándose en 83.109 millones de euros, para estancarse y empezar a disminuir a partir de 2011, a pesar de que el número de empresas siguió creciendo hasta 2014, si se compara con el Gráfico 1.

Los fondos propios, mientras a finales de 2001 alcanzan los 6.096 millones de euros, en 2016 se sitúan en 15.360 millones de euros, lo que supone una variación del 152\%. Debe resaltarse los indicadores de los años 2007 y 2011, pues los fondos propios aumentaron un $189,7 \%$ en el primer caso, y se redujeron un 42,98\% en el segundo, en ambos casos con respecto al ejercicio precedente.

Siguiendo con el Gráfico 2, el pasivo parece mostrar una tendencia similar a la experimentada por los activos, si bien en diferente cuantía. En 2001 su importe total ascendía 9.148 millones de euros, quedando al final del periodo de estudio en 42.537 millones de euros, siendo la variación del 365\%. Si se compara con la evolución de los fondos propios, parece que la trayectoria de las empresas del sector se ha basado más en el 
endeudamiento que en financiación y autofinanciación propias. Las mayores cifras se alcanzan no en un año solo, sino en tres (2010-2011 y 2012).

Gráfico 2. Evolución del activo, pasivo y patrimonio neto

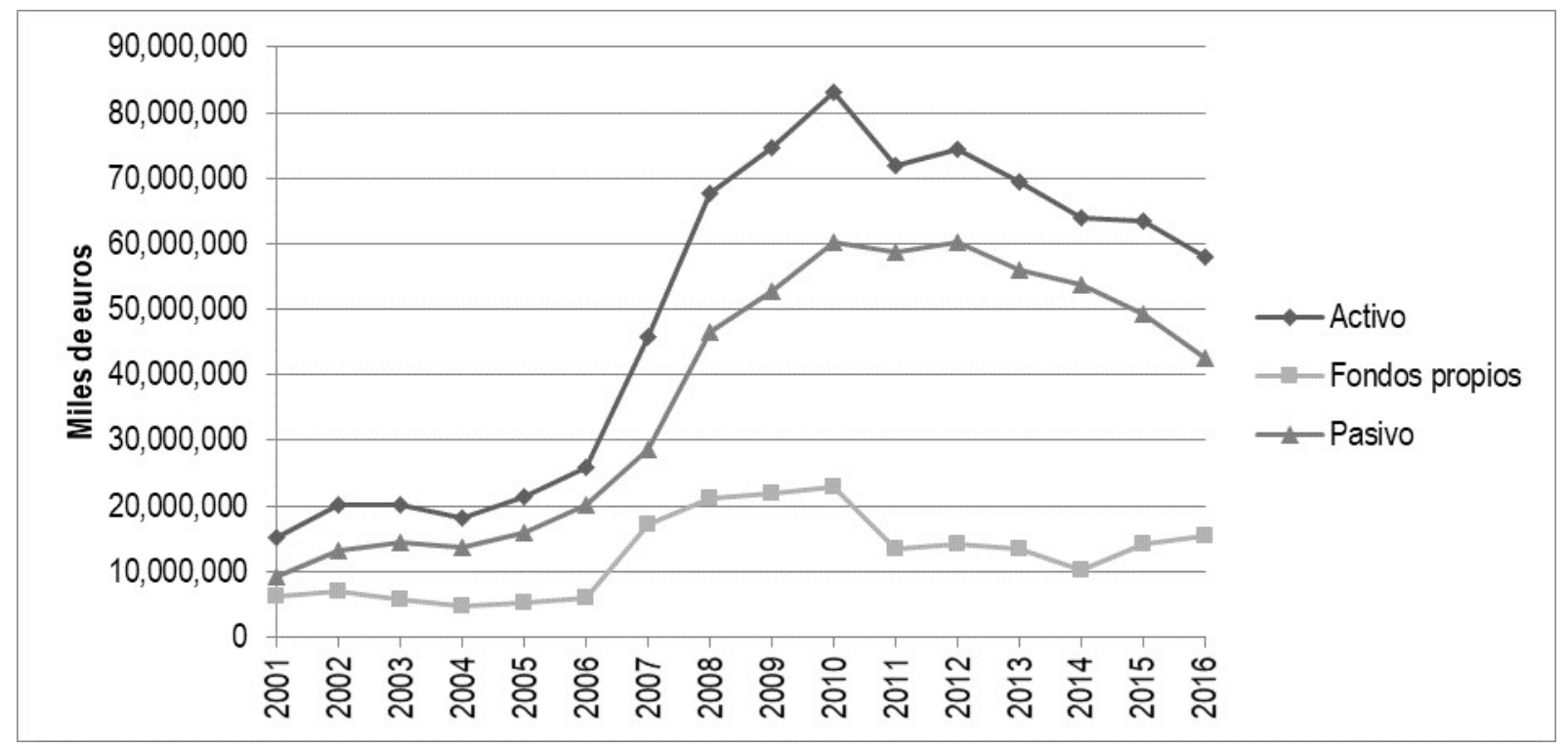

Fuente: Elaboración propia a partir de SABI @ , 2017.

Por otra parte, y como se muestra en el Gráfico 3, a principios del horizonte temporal los ingresos de explotación globales fueron de 2.920 millones de euros, situándose a finales de 2016 en la cota de los 10.958 millones de euros, lo que supone una variación del 275,3\%, siendo en 2008 cuando se obtuvo el nivel más alto de ingresos, 15.904 millones de euros. Cabe destacar que desde 2001, los ingresos mantuvieron una tendencia creciente, hasta que en 2009 caen un 15,75\%, con respecto al ejercicio precedente, siendo la mayor caída de ingresos en el periodo de análisis; manteniéndose esa situación de ahí en adelante.

A pesar del elevado volumen de ingresos de explotación, el resultado de explotación ha sido más bien escaso, lo que denota los elevados costes que se soportan en el sector. En términos relativos, los márgenes más elevados se encuentran en 2005, con un 23,61\% sobre los ingresos de explotación y 2009, con un 24,06\%; mientras que el valor más bajo se alcanza en 2013 con un 6,22\%. En cifras absolutas, el máximo valor se alcanza en 2009, con 3.223 millones de euros, destacando las caídas experimentadas en 2010 (49\% con respecto al año anterior) al obtenerse 1.646 millones de euros y en 2013 al bajar hasta 833 millones de euros (un $58,33 \%$ con respecto al ejercicio precedente).

Lo más destacable de los gastos financieros es que entre 2010 y 2014 sus cifras rebasan el montante del resultado de explotación, siendo en 2013 cuando se alcanza el mayor porcentaje respecto de los ingresos de explotación (21,24\%), en cifras absolutas, 2.847 millones de euros. En 2015 y 2016 mantienen casi el mismo peso relativo que el resultado de explotación, en 
torno al $20 \%$.

Para finalizar, cabe mencionar el resultado contable del sector. Al comienzo de la serie temporal fue de 536 millones de euros, mientras que a finales del periodo se situaron en 586 millones de euros, lo que supone una estabilización del mismo, a pesar del mayor número de empresas en el mercado y las cifras de actividad en ambas fechas, existiendo pérdidas continuadas en el intervalo 2011-2014. El año en que se obtuvo el mejor margen respecto de los ingresos de explotación fue en 2002 (32,44\%), mientras que el peor fue en 2013 (8,48\%). En el Gráfico 3 se muestra la tendencia observada en cada caso.

Gráfico 3. Evolución de los ingresos de explotación, resultado de explotación, gastos financieros y resultado contable

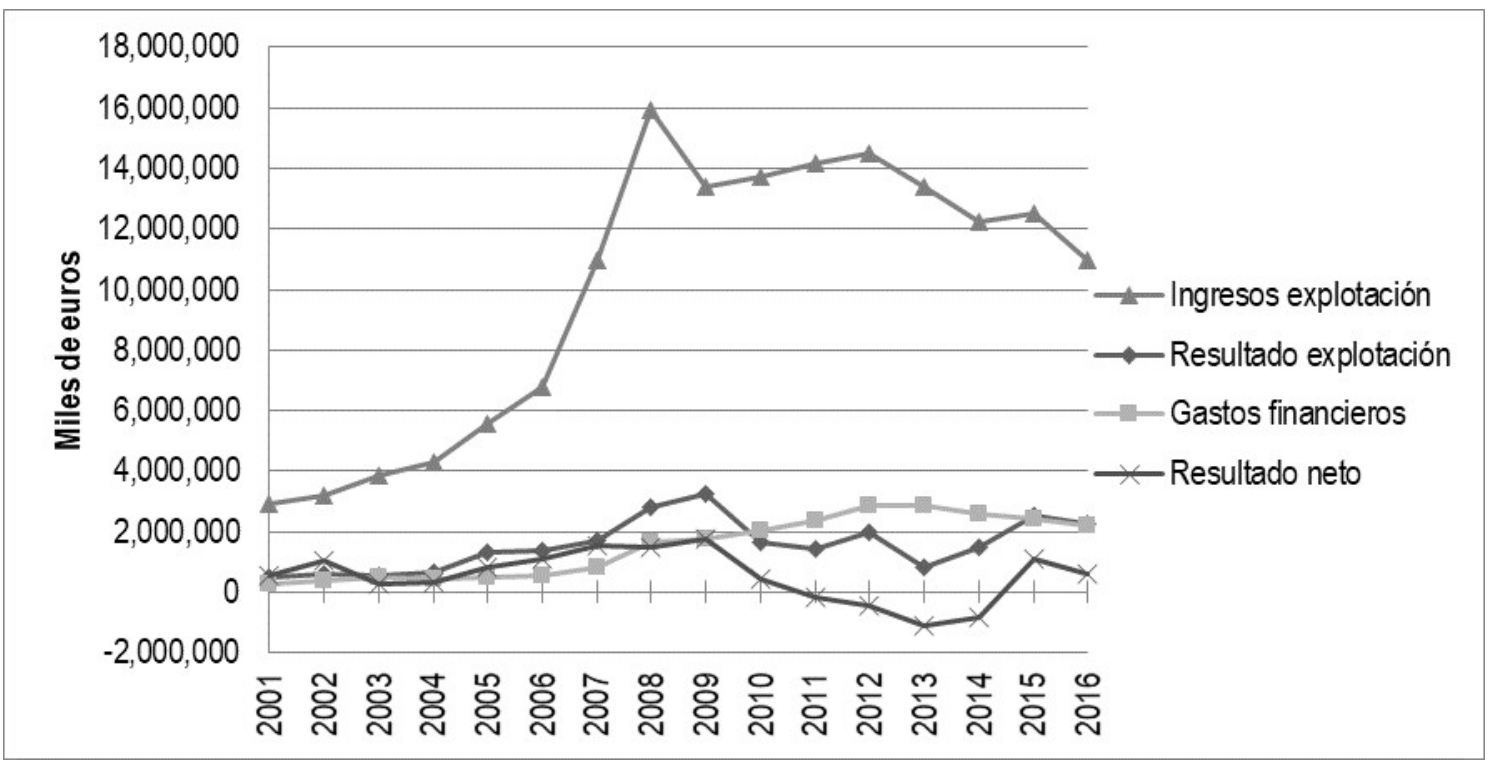

Fuente: Elaboración propia a partir de SABI @ , 2017.

Por otra parte, en la Tabla 2 se exponen los valores en 2001, 2014 y 2016 de las diferentes ratios consideradas, destacando la existencia de una rentabilidad financiera media negativa en 2014 y 2016, en contraste con la rentabilidad económica, si bien la mediana es positiva. También las ratios de solvencia y endeudamiento son muy elevadas.

Tabla 2. Estadística descriptiva básica 2001, 2014 y 2016

\begin{tabular}{ccrrr}
\hline Ratio & N & Media & Mediana & Percentil 75 \\
\hline Rentabilidad financiera 2001 & 792 & 0,0137 & 0,0319 & 0,1762 \\
Rentabilidad financiera 2014 & 7.793 & $-0,3285$ & 0,0019 & 0,1377
\end{tabular}


Tabla 2. Continuación...

\begin{tabular}{lrrrr}
\hline \multicolumn{1}{c}{ Ratio } & \multicolumn{1}{c}{ N } & Media & Mediana & Percentil 75 \\
\hline Rentabilidad financiera 2016 & 7.428 & $-0,4208$ & 0,0367 & 0,1892 \\
Rentabilidad económica 2001 & 792 & 0,0335 & 0,0317 & 0,0754 \\
Rentabilidad económica 2014 & 7.793 & 0,0535 & 0,0161 & 0,0464 \\
Rentabilidad económica 2016 & 7.428 & 0,0195 & 0,0269 & 0,0563 \\
Solvencia 2001 & 741 & 5,9989 & 1,108 & 2,359 \\
Solvencia 2014 & 7.565 & 4,8322 & 1,136 & 2,0857 \\
Solvencia 2016 & 7.271 & 5,1821 & 1,3378 & 2,6025 \\
Endeudamiento 2001 & 792 & 27,1447 & 1,9423 & 6,3765 \\
Endeudamiento 2014 & 7.793 & 24,1084 & 3,2545 & 9,8258 \\
Endeudamiento 2016 & 7.428 & 67,9779 & 2,4686 & 7,6484 \\
\hline
\end{tabular}

Las rentabilidades se presentan en tanto por uno. Las demás ratios se presentan como proporciones

Fuente: Elaboración propia.

\section{¿Ha tenido algún impacto la aprobación del Real Decreto 661/2007?}

En 2007 se aprueba el Real Decreto 661/2007, con el propósito principal de fomentar la producción de energía con fuentes renovables. Para ello se introdujo la figura de las primas al Régimen Especial. Además, aquellos operadores que se acogieran a este régimen tendrían garantizado su acceso al sistema eléctrico de forma prioritaria.

En la Tabla 3 se muestran los datos básicos relativos a la rentabilidad, la solvencia y el endeudamiento, en los dos años anteriores (2005-2006) y los dos posteriores
(2008-2009) a la aprobación de esta norma legal. El primer elemento reseñable es el relativo al número de empresas que conforman la muestra, pues entre un periodo y el siguiente su número promedio se duplica. No se incluyen las empresas con fondos propios negativos, cuyas cifras promedio ascendieron a 284, en el periodo previo (11,64\% del total de empresas inscritas en ese intervalo), y 1.558 , en el posterior (23,57\% del total). Las ratios de rentabilidad económica y financiera y de solvencia mejoran, en los tres estadísticos considerados, excepto en el caso de la solvencia media que empeora. Por su parte, el endeudamiento aumenta. 
Tabla 3. Estadística descriptiva de diferentes ratios y la aprobación del RD 661/2007

\begin{tabular}{lcccc}
\hline \multicolumn{1}{c}{ Ratios } & & & & Percentil \\
& N & Media & Mediana & 75 \\
\hline Rentabilidad financiera (antes) & 3.013 & $-0,0232$ & 0,0000 & 0,1066 \\
Rentabilidad financiera (después) & 6.219 & 0,0621 & 0,0296 & 0,3293 \\
Rentabilidad económica (antes) & 3.013 & 0,0199 & 0,0000 & 0,0403 \\
Rentabilidad económica (después) & 6.219 & 0,0311 & 0,0352 & 0,0660 \\
Solvencia (antes) & 2.223 & 9,6325 & 1,1289 & 2,3911 \\
Solvencia (después) & 5.851 & 5,6671 & 1,1888 & 2,5344 \\
Endeudamiento (antes) & 3.013 & 40,1513 & 1,7951 & 8,8456 \\
Endeudamiento (después) & 6.219 & 102,6411 & 5,7220 & 22,2033 \\
\hline
\end{tabular}

Fuente: Elaboración propia.

Si el estudio se centra en las empresas registradas tanto antes y como después de la reforma legal (empresas comunes), salvo la solvencia y el endeudamiento (en ambos casos, en la mediana y el percentil 75), el resto de indicadores experimenta una mejoría (Tabla 4).

Tabla 4. Estadística descriptiva de diferentes ratios y la aprobación del RD 661/2007 (empresas comunes)

\begin{tabular}{lcccc}
\hline \multicolumn{1}{c}{ Ratios } & N & Media & Mediana & Percentil \\
\hline Rentabilidad financiera (antes) & 1.938 & $-0,0107$ & 0,0000 & 0,1730 \\
Rentabilidad financiera (después) & 1.938 & 0,0584 & 0,0592 & 0,2563 \\
Rentabilidad económica (antes) & 1.938 & 0,0386 & 0,0041 & 0,0630 \\
Rentabilidad económica (después) & 1.938 & 0,0599 & 0,0439 & 0,0875 \\
\hline
\end{tabular}


Tabla 4. Continuación...

\begin{tabular}{lcrrr}
\hline \multicolumn{1}{c}{ Ratios } & N & Media & Mediana & Percentil \\
\hline Solvencia (antes) & 1.595 & 11,5432 & 1,2072 & 2,7512 \\
Solvencia (después) & 1.595 & 7,3469 & 1,1883 & 2,6666 \\
Endeudamiento (antes) & 1.938 & 41,2949 & 2,4780 & 8,5107 \\
Endeudamiento (después) & 1.938 & 36,7303 & 3,6488 & 10,3962 \\
\hline
\end{tabular}

Fuente: Elaboración propia.

En la Tabla 5 se presentan los resultados del análisis estadístico para muestras pareadas en empresas comunes utilizando el test de Wilcoxon-Mann-Whitney ${ }^{1}$. Los cambios producidos en la rentabilidad económica y el endeudamiento son estadísticamente significativos para un $\alpha=0,01$, mientras que las variaciones producidas en la rentabilidad financiera, lo son para un $\alpha=0,05$. En el caso de la solvencia no parecen ser significativas. Es factible suponer que las primas al régimen especial hayan contribuido a mejorar los resultados de las empresas del sector, pero también a aumentar el endeudamiento por la adquisición y/o ampliación de instalaciones.

Tabla 5. Relación estadística entre determinadas magnitudes contables y la aprobación del Real Decreto 661/2007

\begin{tabular}{lccc}
\hline \multicolumn{1}{c}{ Variable } & $\mathbf{N}$ & Estadístico & p-value \\
\hline Rentabilidad financiera & 1.938 & $-1,907$ & $0,0565^{* *}$ \\
Rentabilidad económica & 1.938 & $-16,664$ & $0,0000^{* * *}$ \\
Ratio de solvencia & 1.595 & $-0,901$ & 0,3678
\end{tabular}

\footnotetext{
${ }^{1}$ Se optó por un test no paramétrico, tras rechazarse la hipótesis de normalidad al realizar las pruebas de asimetría, curtosis y Shapiro-Wilks en la aplicación seleccionada. 
Tabla 5. Continuación...

\begin{tabular}{lccc}
\hline \multicolumn{1}{c}{ Variable } & N & Estadístico & p-value \\
\hline Ratio de endeudamiento & 1.938 & $-2,393$ & $0,0167^{* * *}$ \\
\hline Test utilizado: Wilcoxon-Mann-Whitney para muestras pareadas & & \\
\hline$*, * *, * * *$ : nivel de significación del 10\%, 5\% y 1\%, respectivamente & \\
\hline
\end{tabular}

Fuente: Elaboración propia.

Finalmente, si se hace un estudio de los cambios producidos en el signo de la rentabilidad financiera en las empresas comunes, se observa que 497 empresas mantuvieron rentabilidades negativas de un periodo al siguiente frente a 713 que continuaron con rentabilidades positivas. Sin embargo, 531 pasaron de tener rentabilidad negativa a ser positiva (51,65\% de las empresas con rendimientos negativos en el periodo previo), mientras que 197 pasaron de tener valores positivos a negativos (21,64\% del total de empresas con rendimientos positivos en el periodo previo). Aplicando la prueba $\chi^{2}$ de Pearson, para un grado de libertad se obtuvo un valor de 149,6802 (p-value $=0,000$ ), por lo que se considera que los cambios han sido significativos.

Desde la perspectiva de la rentabilidad económica, el estudio de los cambios acaecidos en su signo parece más llamativo: 181 empresas siguieron con rendimientos negativos de un periodo al siguiente en comparación con 964 que continuaron con rentabilidades positivas. 674 compañías pasaron de tener rentabilidad negativa a ser positiva $(78,83 \%$ de las empresas con rendimientos negativos en el periodo previo), mientras que 119 pasaron de tener valores positivos a negativos $(10,98 \%$ del total de empresas con rendimientos positivos en el periodo previo). Aparentemente las medidas adoptadas han permitido mejorar la rentabilidad económica de un importante número de empresas del sector. Aplicando la prueba $\chi^{2}$ de Pearson, para un grado de libertad se obtuvo un valor de 37,8569 (p-value $=0,000$ ), ello quiere decir que los cambios han sido significativos.

\section{¿Qué han supuesto los cambios normativos de 2013?}

En 2013 se aprueban varias normas legales de calado con el propósito declarado de hacer frente al déficit de tarifa. Son los casos del Real Decreto-ley 2/2013, que fija un nuevo método de cálculo de las retribuciones, tarifas y primas, con el objetivo de reducir los costes y evitar que los consumidores asuman dicha carga; el Real Decreto-ley 9/2013, que suprime de facto el régimen especial, habilitando al Gobierno a aprobar un nuevo marco jurídico y económico para las instalaciones de producción de energía eléctrica existentes a partir de energía renovable, cogeneración y residuos; y la Ley 24/2013, que entre otros aspectos establece que, con carácter general, las instalaciones de autoconsumo estarán obligadas a contribuir a la financiación de los costes y servicios del sistema en la misma cuantía que el resto de los consumidores, previéndose determinadas excepciones.

En la Tabla 6 se presentan, al igual que en el estudio anterior, los datos básicos relativos a la rentabilidad, la solvencia y el endeudamiento, en los dos años anteriores (en este caso 2011-2012) y los dos posteriores (20142015) a la reforma de 2013. En términos generales y 
para todas las empresas registradas, los indicadores mediana de la rentabilidad económica que empeoran. mejoran, salvo la rentabilidad financiera media y la

Tabla 6. Estadística descriptiva de diferentes ratios y los cambios normativos de 2013

\begin{tabular}{lcccc}
\hline \multicolumn{1}{c}{ Ratios } & & & & Percentil \\
& N & Media & Mediana & 75 \\
\hline Rentabilidad financiera (antes) & 8.107 & $-0,1013$ & 0,0091 & 0,1332 \\
Rentabilidad financiera (después) & 8.592 & $-0,1272$ & 0,0321 & 0,1938 \\
Rentabilidad económica (antes) & 8.107 & 0,0290 & 0,0305 & 0,0487 \\
Rentabilidad económica (después) & 8.592 & 0,0473 & 0,0279 & 0,0544 \\
Solvencia (antes) & 7.830 & 5,2272 & 1,2188 & 2,3612 \\
Solvencia (después) & 8.447 & 5,3041 & 1,2062 & 2,3536 \\
Endeudamiento (antes) & 8.107 & 44,5112 & 3,8185 & 13,6312 \\
Endeudamiento (después) & 8.592 & 25,0876 & 3,5939 & 10,5698 \\
\hline
\end{tabular}

Fuente: Elaboración propia.

Asimismo, en promedio, el número de empresas con fondos propios negativos en el periodo previo es de 2.141 (23,05\% del total de empresas inscritas) mientras que en el posterior es de 2.957 (27,62\% del total). ambos periodos, en la Tabla 7 se observa que, a diferencia de los datos registrados en la tabla precedente, la rentabilidad financiera promedio pasa de tener valores positivos a negativos.

Si solo se tienen en cuenta las empresas comunes a

Tabla 7. Estadística descriptiva de diferentes ratios y los cambios de 2013, empresas comunes

\begin{tabular}{ccrrrr}
\hline Ratios & & & & Percentil \\
& N & Media & Mediana & 75 \\
\hline Rentabilidad financiera (antes) & 6.458 & 0,046 & 0,0221 & 0,1382
\end{tabular}


Tabla 7. Continuación...

\begin{tabular}{lcrrr}
\hline \multicolumn{1}{c}{ Ratios } & \multicolumn{1}{c}{ N } & Media & Mediana & Percentil \\
\hline Rentabilidad financiera (después) & 6.458 & $-0,3079$ & 0,0333 & 0,1950 \\
Rentabilidad económica (antes) & 6.458 & 0,0359 & 0,0313 & 0,0499 \\
Rentabilidad económica (después) & 6.458 & 0,0552 & 0,0287 & 0,0567 \\
Solvencia (antes) & 6.217 & 5,2567 & 1,2955 & 2,6312 \\
Solvencia (después) & 6.217 & 5,125 & 1,2454 & 2,2260 \\
Endeudamiento (antes) & 6.458 & 32,5226 & 3,2125 & 10,178 \\
Endeudamiento (después) & 6.458 & 21,5514 & 3,2524 & 8,5410 \\
\hline
\end{tabular}

Fuente: Elaboración propia.

De acuerdo con los datos registrados en la Tabla 8, los cambios producidos son estadísticamente significativos para un $\alpha=0,01$, excepto en el caso de la rentabilidad financiera, cuyas variaciones no parecen ser significativas. Es decir, en el caso de las empresas comunes una regulación más restrictiva no parece haberles afectado negativamente en lo que respecta a la rentabilidad financiera.

Tabla 8. Relación estadística entre determinadas magnitudes contables y los cambios de 2013

\begin{tabular}{lccc}
\hline \multicolumn{1}{c}{ Variable } & N & Estadístico & p-value \\
\hline Rentabilidad financiera & 6.458 & $-0,051$ & 0,9595 \\
Rentabilidad económica & 6.458 & 3,855 & $0,0001^{* * *}$ \\
Ratio de solvencia & 6.217 & 6,741 & $0,0000^{* * *}$ \\
Ratio de endeudamiento & 6.458 & 14,222 & $0,0000^{* * *}$ \\
\hline Test utilizado: Wilcoxon-Mann-Whitney para muestras pareadas & & \\
\hline$*, * *, * * *:$ nivel de significación del 10\%, 5\% y 1\%, respectivamente & \\
\hline
\end{tabular}

Fuente: Elaboración propia. 
Si se compara el signo de las rentabilidades financieras de las empresas comunes, antes y después de las reformas legales de 2013, las diferencias son estadísticamente significativas, aplicando la prueba $\chi^{2}$ de Pearson, para un grado de libertad (el valor fue de 311,1328, p-value = 0,000). Así, 1.090 pasaron de poseer una rentabilidad financiera positiva antes de los cambios de 2013 a negativa en el periodo posterior (el 28,98\% de las empresas con rentabilidad positiva en el periodo previo), mientras que 1.333 pasaron de cifras negativas a positivas (el 49,40\% de las empresas con valores negativos en el periodo precedente). El resto de entidades siguió igual (1.365 continuaron con rendimientos negativos y 2.670 con valores positivos).

En cuanto al signo de la rentabilidad económica, también las diferencias son estadísticamente significativas, si se atiene a la prueba $\chi^{2}$ de Pearson, para un grado de libertad (el valor fue de 424,2857, pvalue $=0,000)$. En este sentido, 807 empresas pasaron de poseer una rentabilidad positiva antes de los cambios de 2013 a negativa en el periodo posterior (el 15,83\% de las compañías con rentabilidad positiva en ese periodo), mientras que 795 pasaron de cifras negativas a positivas (el 58,45\% de las empresas con valores negativos en el periodo precedente). El resto de entidades siguió igual (565 continuaron con rendimientos negativos y 4.291 con valores positivos).

\section{Conclusiones}

El objetivo del presente trabajo ha sido obtener evidencias acerca de una posible relación entre determinadas magnitudes contables del sector de producción de energías renovables en España y la actividad regulatoria.

A tal objeto se partió de una muestra de 14.509 empresas, dedicadas en exclusiva a la producción de electricidad por medios renovables (solar, eólica e hidráulica), que declararon ingresos en algún momento del periodo de análisis, el cual abarcó de 2001 a 2016, partiendo de la información recopilada en la base de datos SABI (C) (2017), propiedad de la compañía Informa, S.A.

Entre las principales conclusiones destaca el crecimiento, en volumen de activos, del sector de producción de energías renovables entre los años 2005 y 2009, tras la aprobación del Plan de Energías Renovables 2005-2010 y el Real Decreto 661/2007, para estancarse en 2010. Por otro lado, cabe reseñar el espectacular incremento en el número de empresas inscritas, pasando de 881 en 2001 a 9.913 en 2016, alcanzando su máximo en 2014 con 11.011 compañías.

Además, y en términos generales, los resultados del sector han sido bajos, llegando a pérdidas en determinados momentos, como sucedió entre 2011 y 2014, si bien fue en 2007 cuando se alcanzó el mayor porcentaje de empresas con pérdidas (62,71\%). En lo que respecta a empresas con fondos propios negativos, 2013 fue el peor año, al alcanzarse un 30,39\% del total de empresas inscritas.

En cuanto a la producción normativa, se analizó la emisión del Real Decreto 661/2007, gracias al cual se implantaron las primas al régimen especial de producción de energía bajo fuentes renovables; así como la aprobación del Real Decreto-ley 2/2013, el Real Decreto-ley 9/2013, y la Ley 24/2013, que se tradujeron en la práctica, en la desaparición de facto de dicho régimen.

En el primer supuesto sólo fueron significativos el aumento en la rentabilidad económica y la reducción en el endeudamiento, del periodo previo a la aprobación del Real Decreto 661/2007 al posterior, no así en el caso del incremento de la rentabilidad financiera $y$ la disminución de la solvencia. 
Sin embargo y en lo que respecta a los cambios normativos de 2013, tanto la disminución de la rentabilidad financiera, como de la solvencia y el incremento del endeudamiento fueron estadísticamente significativos, pero no en el caso de la reducción de la rentabilidad económica.

En resumen, de todo lo expuesto cabe presumir que hay relación entre la emisión de normas legales y los cambios producidos en determinadas magnitudes contables las empresas del sector de producción de electricidad mediante fuentes de energía renovable.

\section{Referencias:}

Agencia Estatal de Evaluación de las Políticas Públicas y la Calidad de los Servicios (2011). Evaluación del Plan Español de Energías Renovables 2005-2010. Ministerio de Política Territorial y Administración Pública, Gobierno de España. Recuperado de http://www.aeval.es/export/sites/aeval/comun/pdf/eval uaciones/E24-PER.pdf

Aragonés, V.; Barquín, J.; Alba, J. (2016). The new Spanish self-consumption regulation. Energy Procedia, 106, pp. 245-257.

https://dx.doi.org/10.1016/j.egypro.2016.12.120

Comisión Nacional de la Energía (2009). Memoria 2008. Madrid. Recuperado de https://www.cnmc.es/file/30838/download

Couture, T.D; Cory, K.; Kreycik, C.; Williams, E. (2010). A Policymaker's Guide to Feed-in Tariff Policy Design. Technical Report NREL/TP-6A2-44849 July 2010, National Renewable Energy Laboratory. Recuperado de

https://www.nrel.gov/docs/fy10osti/44849.pdf
Directiva 2001/77/CE del Parlamento Europeo y del Consejo, de 27 de septiembre de 2001 (DO L 283 de 27.10.2001), relativa a la promoción de la electricidad generada a partir de fuentes de energía renovables en el mercado interior de la electricidad.

Directiva 2003/30/CE del Parlamento Europeo y del Consejo de 8 de mayo de 2003 (DOUE de 17.5.2003 L123/42), relativa al fomento del uso de biocarburantes u otros combustibles renovables en el transporte.

Directiva 2009/28/CE del Parlamento Europeo y del Consejo de 23 de abril de 2009 (DOUE 5.6.2009 L 140/6), relativa al fomento del uso de energía procedente de fuentes renovables y por la que se modifican y se derogan las Directivas 2001/77/CE y 2003/30/CE.

España pierde su primer arbitraje internacional por los recortes a las renovables. (2017, 05 de mayo). Público. Recuperado de https://www.publico.es/economia/espana-pierdeprimer-arbitraje-internacional.html

España sigue su racha y gana otro laudo renovable (132 millones) por unanimidad, (2020, 11 de marzo). El Confidencial. Recuperado de https://www.elconfidencial.com/empresas/2020-0311/espana-racha-gana-otra-laudo-renovableunanimidad_2490988/

Fouquet, D.; Nysten, J.V. (2015). Policy Briefing. Retroactive and retrospective changes and moratoria to RES support. Keep On Track 2020 - EUFORES European Forum for Renewable Energy Sources. Recuperado de http://www.keepontrack.eu/contents/publicationsbiann ualnationalpolicyupdatesversions/policy-briefing6retroactive-and-retrospective-changes-and-moratoriato-res-support.pdf 
Hair, J.F.; Anderson, R.E.; Tatham, R.l.; Black, W.C. (1999). Análisis multivariante (5a.ed). Madrid: Prentice Hall Iberia.

Instituto para la Diversificación y Ahorro de la Energía (1999). Plan de fomento de las energías renovables en España. Ministerio de Ciencia y Tecnología, Gobierno de España. Recuperado de http://www.idae.es/uploads/documentos/documentos_4 044_PFER2000-10_1999_1cd4b316.pdf

Instituto para la Diversificación y Ahorro de la Energía (2005). Plan de energías renovables en España 20052010. Ministerio de Industria, Turismo y Comercio, Gobierno de España. Recuperado de http://www.idae.es/uploads/documentos/documentos_P ER_2005-2010_8_de_gosto-

2005_Completo.(modificacionpag_63)_Copia_2_3012 54a0.pdf

Instituto para la Diversificación y Ahorro Energético (2011). Plan de energías renovables 2011-2020. Ministerio de Industria, Turismo y Comercio, Gobierno de España. Recuperado de http://www.idae.es/tecnologias/energiasrenovables/plan-de-energias-renovables-2011-2020

Jaraitė, J.; Kažukauskas, A. (2013). The profitability of electricity generating firms and policies promoting renewable energy. Energy Economics, 40, pp.858-865. https://dx.doi.org/10.1016/j.eneco.2013.10.001

Jovell, A.J. (1995). Análisis de regresión logística. Cuadernos metodológicos 15. Madrid: Centro de Investigaciones Sociológicas.

Ley 54/1997, de 27 de noviembre (BOE de 28 de noviembre), del Sector Eléctrico.
Ley 2/2011, de 4 de marzo (BOE de 5 de marzo), de Economía Sostenible.

Ley 15/2012, de 27 de diciembre (BOE de 28 de diciembre), de medidas fiscales para la sostenibilidad energética.

Ley 24/2013, de 26 de diciembre (BOE de 27 de diciembre), del Sector Eléctrico.

Lomas, J.C.; Muñoz-Cerón, E.; Nofuentes, G.; de la Casa, J. (2018). Sale of profitable but unaffordable PV plants in Spain: Analysis of a real case. Energy Policy, 117, pp.279-294.

https://doi.org/10.1016/j.enpol.2018.03.014

López Prol, J. (2018). Regulation, profitability and diffusion of photovoltaic grid-connected systems: A comparative analysis of Germany and Spain. Renewable and Sustainable Energy Reviews, 91, pp. 1170-1181. https://doi.org/10.1016/j.rser.2018.04.030

Mesa, M. (2015, 18 de febrero). Dos asociaciones canarias denuncian ante Bruselas la "crítica" situación de las renovables en el Archipiélago. Canarias Ahora. Recuperado de https://www.eldiario.es/canariasahora/energia/asociaci ones-Bruselas-situacion-renovables-

Archipielago_0_358015348.html

Mir-Artigues, P.; Cerdá, E.; del Rio, P. (2015). Analyzing the impact of cost-containment mechanisms on the profitability of solar PV plants in Spain. Renewable and Sustainable Energy Reviews, 46, pp.166-177.

https://dx.doi.org/10.1016/j.rser.2015.02.005

Montoya, F.G.; Aguilera, M.J.; Manzano-Agugliaro, F. (2014). Renewable energy production in Spain: A review. Renewable and Sustainable Energy Reviews, 33, pp.509-531.

https://dx.doi.org/10.1016/j.rser.2014.01.091 
Noceda, M.A. (2017, 05 de mayo). España pierde su primer arbitraje internacional ante al Ciadi por los recortes en las renovables. El País. Recuperado de https://elpais.com/economia/2017/05/05/actualidad/149 3976214_856666.html

Orden IET/1045/2014, de 16 de junio (BOE de 20 de junio), por la que se aprueban los parámetros retributivos de las instalaciones tipo aplicables a determinadas instalaciones de producción de energía eléctrica a partir de fuentes de energía renovables, cogeneración y residuos.

Real Decreto 2818/1998, de 23 de diciembre (BOE de 30 de diciembre), sobre producción de energía eléctrica por instalaciones abastecidas por recursos o fuentes de energía renovables, residuos y cogeneración.

Real Decreto 475/2007, de 13 de abril (BOE de 28 de abril), por el que se aprueba la Clasificación Nacional de Actividades Económicas 2009 (CNAE-2009).

Real Decreto 661/2007, de 25 de mayo (BOE de 26 de mayo), por el que se regula la actividad de producción de energía eléctrica en régimen especial.

Real Decreto 1028/2007, de 20 de julio (BOE de 1 de agosto), por el que se establece el procedimiento administrativo para la tramitación de las solicitudes de autorización de instalaciones de generación eléctrica en el mar territorial.

Real Decreto 1003/2010, de 5 de agosto (BOE de 6 de agosto), por el que se regula la liquidación de la prima equivalente a las instalaciones de producción de energía eléctrica de tecnología fotovoltaica en régimen especial.

Real Decreto 413/2014, de 6 de junio (BOE de 10 de junio), por el que se regula la actividad de producción de energía eléctrica a partir de fuentes de energía renovables, cogeneración y residuos.
Real Decreto 900/2015, de 9 de octubre (BOE de 10 de octubre), por el que se regulan las condiciones administrativas, técnicas y económicas de las modalidades de suministro de energía eléctrica con autoconsumo y de producción con autoconsumo.

Real Decreto-ley 6/2009, de 30 de abril (BOE de 7 de mayo), por el que se adoptan determinadas medidas en el sector energético y se aprueba el bono social.

Real Decreto-ley 14/2010, de 23 de diciembre (BOE de 24 de diciembre), por el que se establecen medidas urgentes para la corrección del déficit tarifario del sector eléctrico.

Real Decreto-ley 1/2012, de 27 de enero (BOE de 28 de enero), por el que se procede a la suspensión de los procedimientos de preasignación de retribución y a la supresión de los incentivos económicos para nuevas instalaciones de producción de energía eléctrica a partir de cogeneración, fuentes de energía renovables y residuos.

Real Decreto-ley 2/2013, de 1 de febrero (BOE de 2 de febrero), de medidas urgentes en el sistema eléctrico y en el sector financiero.

Real Decreto-ley 9/2013, de 12 de julio (BOE de 13 de julio), por el que se adoptan medidas urgentes para garantizar la estabilidad financiera del sistema eléctrico.

Real Decreto-ley 9/2015, de 10 de julio (BOE de 11 de julio), de medidas urgentes para reducir la carga tributaria soportada por los contribuyentes del Impuesto sobre la Renta de las Personas Físicas y otras medidas de carácter económico.

SABI. (2017). Sistema de Análisis de Balances Ibéricos. Informa. Recuperado de https://www.einforma.com/soluciones-yherramientas/sabi 
Secretaría de Estado de Energía (2017). La energía en España 2016. Ministerio de Energía, Turismo y Agenda Digital, Gobierno de España. Recuperado de https://www.mincotur.gob.es/energia/balances/Balance s/LibrosEnergia/energia-espana-2016.pdf

StataCorp (2009). Stata Statistical Software: Release 11. College Station, TX: StataCorp LP.

Tribunal Supremo (2016a). Planteamiento de cuestión de inconstitucionalidad.- Artículos 1, 4.1, 6.1 y 8 de la Ley 15/2012, de 27 de diciembre, de medidas fiscales para la sostenibilidad energética, que regulan el impuesto sobre el valor de la producción de la energía eléctrica, por su eventual vulneración del principio de capacidad económica proclamado en el artículo 31.1 de la Constitución. Sala de lo ContenciosoAdministrativo, Sección Segunda, Recurso de Casación, 14/06/2016, Recurso 2554/2014.
Tribunal Supremo (2016b). Planteamiento de cuestión de inconstitucionalidad.- Artículos 1, 4.1, 6.1 y 8 de la Ley 15/2012, de 27 de diciembre, de medidas fiscales para la sostenibilidad energética, que regulan el impuesto sobre el valor de la producción de la energía eléctrica, por su eventual vulneración del principio de capacidad económica proclamado en el artículo 31.1 de la Constitución. Sala de lo ContenciosoAdministrativo, Sección Segunda, Recurso de Casación, 14/06/2016, Recurso 2955/2014. 